\title{
Changes of microbial and metabolome of the equine hindgut during oligofructose- induced laminitis
}

\author{
Maimaiti Tuniyazi ${ }^{\dagger}$, Junying $\mathrm{He}^{\dagger}$, Jian Guo, Shuang Li, Naisheng Zhang, Xiaoyu Hu ${ }^{*}$ and Yunhe Fu* (D)
}

\begin{abstract}
Background: Laminitis is a common and serve disease which caused by inflammation and pathological changes of the laminar junction. However, the pathologic mechanism remains unclear. In this study we aimed to investigate changes of the gut microbiota and metabolomics in oligofructose-induced laminitis of horses.

Results: Animals submitted to treatment with oligofructose had lower fecal pH but higher lactic acid, histamine, and Lipopolysaccharide (LPS) in serum. Meanwhile, oligofructose altered composition of the hindgut bacterial community, demonstrated by increasing relative abundance of Lactobacillus and Megasphaera. In addition, the metabolome analysis revealed that treatment with oligofructose decreased 84 metabolites while 53 metabolites increased, such as dihydrothymine, N3,N4-Dimethyl-L-arginine, 10E,12Z-Octadecadienoic acid, and asparagine. Pathway analysis revealed that aldosterone synthesis and secretion, regulation of lipolysis in adipocytes, steroid hormone biosynthesis, pyrimidine metabolism, biosynthesis of unsaturated fatty acids, and galactose metabolism were significantly different between healthy and laminitis horses. Furthermore, correlation analysis between gut microbiota and metabolites indicated that Lactobacillus and/or Megasphaera were positively associated with the dihydrothymine, N3,N4-Dimethyl-L-arginine, 10E,12Z-Octadecadienoic acid, and asparagine.
\end{abstract}

Conclusions: These results revealed that disturbance of gut microbiota and changes of metabolites were occurred during the development of equine laminitis, and these results may provide novel insights to detect biomarkers for a better understanding of the potential mechanism and prevention strategies for laminitis in horses.

Keywords: Equine, Laminitis, Gut microbiota, Metabolomics

\section{Background}

The domestication of horses occurred 5000-6000 years ago and has become an important aid for humans in battle, transportation and trade throughout the history [1]. Now horses are used for their specific qualities such as speed and jumping ability in competitions all over the world. Thus, diseases in horses, especially sports related ones, such as laminitis, have been becoming increasingly important and bringing mental, financial, and technical

\footnotetext{
* Correspondence: huxiaoyu@jlu.edu.cn; fuyunhesky@163.com

${ }^{\dagger}$ Tuniyazi Maimaiti and Junying He contributed equally to this work. Department of Clinical Veterinary Medicine, College of Veterinary Medicine, Jilin University, Changchun, Jilin Province 130062, People's Republic of China
}

challenges to horse owners and veterinary clinicians. Recent years our studies in equine laminitis have been focused mainly on gut microbiota, since researcher Lippold S. et al. [2] found that domesticated horses had reduced microbial diversity compared to wild horses, which may be the cause of laminitis.

Laminitis is defined as the loss of attachment between the inner hoof wall and the distal phalanx, which result in the bone to be driven down into the hoof, destroying the surrounding arteries and veins, and finally crushing the dermis and crown of the sole [3]. It is one of the most serious diseases of the equine foot with a total incidences of about $15 \%$ of all lameness in the USA, and 
with over 27 and $4.7 \%$ of cases unable to recover and mortality respectively [4]. Laminitis will occur when the attachment between the distal phalanx and inner hoof wall fails. Evidence showed that a large number of factors are contribute to laminitis, including feeding, injury, obesity, and pregnancy, among them, however, the most common reason is overload of carbohydrate due to exposure to certain pastures or excessive grain-based diets $[5,6]$. Unfortunately, the exact pathophysiological mechanisms of equine laminitis remain unclear.

The horse gut microbiota facilities digestion and nutrient absorption for host energy production, short chain fatty acid production, and immune health such as protecting against pathogens and diseases. Thus, in horses keeping the gut microbial community and metabolites at a normal and stable condition is crucial for defending a large array of both intestinal and non-intestinal diseases [7-9]. Previous studies suggested that development of equine laminitis is associated with disturbance of the hindgut microbiota and metabolites $[6,10]$, and several theories have been proposed to expound the changing relationship of hindgut microbial community and metabolites during severe laminitis cases. These hypotheses indicated that occurrence of the lamellar ischaemia was associated with considerable numbers of hindgutderived vasoactive agents, such as histamines [11], other vasoactive amines [12], or endotoxins, or contributed to the over-production of host enzymes, especially matrix metalloproteinases [13, 14]. Other studies also demonstrated that the development of acute laminitis in horses was pertinent to lactic acidosis resulting from carbohydrate-overloading $[15,16]$. Among these theories, one common shared cause of laminitis aetiology is imbalance of hindgut bacterial community which induced by overloading carbohydrate and then it leads to initiate a sequence of events which ultimately contributes to developing of equine laminitis. Equine laminitis model, traditionally, was established by overloading starch in studies. However, the unacceptable incidences of colic and mortality were frequent in this model [15]. After finding the fructans ( $\beta$-D-fructose polymer with terminal glucose monomers) that could induce onset of laminitis in horses and the symptoms were identical to that induce by starch, thus Pollitt established a laminitis model using commercial fructose polymers (oligofructose) [17].

\section{Methods}

\section{Animals and treatment}

The present study included 10 healthy horses ( 3 males, 7 mares, age: $6.7 \pm 1.06$ ) were purchased form a horse ranch where managed under identical feeding program for the past two years. We also acknowledged that they had not been exposed to any antibiotics or anthelmintic treatment for at least 3 months prior to the experiment. Horses with any digestive diseases or local hoof lesions were excluded in this project. All animals were managed at university stable where they had free access to water and hey for 3 weeks before experiment. The laminitis model was induced by $10 \mathrm{~g} \mathrm{~kg}^{-1}$ body weight of oligofructose using a nasogastric tube $[17,18]$.

The microbial changes may result from a direct or indirect effect of lifestyle, dietary habit, genetic, or other factors that vary between individuals. In order to eliminate adverse effect brought by inter-individual variations, a self-comparative analysis was proposed to reduce selection biases and achieve more reliable results. Thus, in this study, the samples collected before treatment were used as a control group and compared with the following treatment procedures. Fecal samples were collected through rectum post-oligofructose administration (POA) and immediately stored at $-80^{\circ} \mathrm{C}$ until microbiota analysis. Fecal $\mathrm{pH}$ was detected at $4 \mathrm{~h}$ intervals in the $0-24$ $\mathrm{h}$ POA period. Blood samples were collected from jugular vein with EDTA tubes. The horses were euthanized for pathological assessment of the hooves.

\section{Euthanasia}

Three of the horses, one healthy and two laminates, were euthanized for pathological assessment. We used Xylazine-Ketamine composition (IS Abundant Pharmaceutical CO., LTD, Lanzhou China) in the ratios of 1:5 $(0.1 \mathrm{ml} / \mathrm{kg})$ as Pre-euthanasia drug with the rate of 0.5 $1 \mathrm{ml} /$ second into the jugular vein. Then, sodium pentobarbital (Feilong Pharmaceutical CO., LTD, Heilongjiang China) $0.1 \mathrm{ml} / \mathrm{kg}$ was injected via the jugular vein after the veterinary specialist had confirmed that the animals were fully unconscious and completely unable to feel pain which was tested using needle pocked on the surface of ears.

\section{Hematoxylin and eosin (H\&E) and periodic acid-Schiff (PAS) staining}

Lamellar tissues were collected within $1 \mathrm{~h}$ after euthanasia, and sectioned with a band saw as soon as possible. A $10 \times 10 \times 0.5 \mathrm{~mm}$ section was cut from each hoof which included the hoof wall and lamella tissue. Each specimen was divided into 55 -mm-square blocks that were fixed in $4 \%$ formalin for 24 to $72 \mathrm{~h}$ before performing by routine methods and imbedding in paraffin wax. Care was taken to ensure that the blocks of lamellar tissue were always obtained from the same location for all horses. Sections were stained using H\&E and PAS staining methods and then examined under a light microscope according to a previous study [19]. The H\&E sections and PAS sections were used for detection of lamellar lesions and for morphometry, respectively. All 
digital images were taken using image capture software and a microscope-attached camera (Olympus, Japan).

\section{Doppler Ultrasonographic measurement}

In this study, Doppler ultrasonographic measurement was performed following a previous description on the medial digital artery of both healthy and lame horses after $20 \mathrm{~h}$ of induction [20]. In control group, this process was performed without sedating the horses while all legs bearing weight. However, in laminitis group, doppler ultrasonographic measurement was recorded in standing/lying position under sedation (xylazine $2 \mathrm{mg} / \mathrm{kg}$ IM) due to the severe pain. After adjusting and repeating 3 times each, the averaged diameter for volumetric flow calculation and the diameter of the artery were obtained.

\section{LPS concentration detection}

Blood samples were collected at 0 and $24 \mathrm{~h}$ after oligofructose administration. The samples were centrifuged at 14000 g for $30 \mathrm{~min}$ at $4{ }^{\circ} \mathrm{C}$, and the supernatants were transferred into a sterile, depyrogenated glass tube. The concentration of LPS were detected by a chromogenic endpoint assay (Chinese Horseshoe Crab Reagent Manufactory Co.,Ltd., Xiamen, China) with a minimum detection limit of $0.01 \mathrm{EU} / \mathrm{mL}$ according to the manufacturer's instructions.

\section{Lactic acid and histamine concentrations detection}

Blood samples were collected at 0 and $24 \mathrm{~h}$ after oligofructose administration. The samples were centrifuged at $3000 \mathrm{~g}$ for $30 \mathrm{~min}$ at $4{ }^{\circ} \mathrm{C}$, and the serum were collected to detect the concentration of lactic acid and histamine using detection kits according to the manufacturer's instructions (Suzhou Feiya Biological Technology, Suzhou, China).

\section{DNA extraction, Illumina MiSeq sequencing, and bioinformatics analyses}

The genome DNA from feces was extracted by a CTAB/ SDS method. The DNA concentration and purity were detected by $1 \%$ agarose gels, and the $16 \mathrm{~S}$ rRNA was amplified by barcoded primers (16S V4:515F-806R) targeting the V4 region. The PCR reactions were conducted with Phusion ${ }^{\bullet}$ High-Fidelity PCR Master Mix (New England Biolabs). PCR products were mixed in equal ratios and then purified with a Qiagen Gel Extraction Kit (Qiagen, Germany). Sequencing libraries were generated using the TruSeq ${ }^{\circ}$ DNA PCR-Free Sample Preparation Kit (Illumina, USA). The library quality was evaluated by a Qubit@2.0 Fluorometer (Thermo Scientific) and an Agilent Bioanalyzer 2100 system. Finally, the library was sequenced on an Illumina HiSeq 2500 platform, and 250 bp paired-end reads were generated. Bacterial community diversity and richness were analyzed by ace, chao 1 , the shannon index, the simpson index and the observed species. The distance of bacterial community between control and laminitis was evaluated by nonmetric multidimensional scaling (NMDS) of Bray-Curitis dissimilarity. The bacterial taxa differentially between control and laminitis was evaluated by linear discriminant analysis (LDA) effect size (LEfSe), and the Venn diagrams was conducted to evaluate the numbers of core genera in the cecal contents from the control and the laminitis group horse.

\section{Metabolic extractions}

Intestinal contents were resuspended with prechilled $80 \%$ methanol and was incubated $1 \mathrm{~h}$ at $-20^{\circ} \mathrm{C}$. Then, the samples were centrifuged $14,000 \mathrm{~g}$ for $20 \mathrm{~min}$ at $4{ }^{\circ} \mathrm{C}$. The supernatants were transferred to a fresh Eppendorf tube and spun in a vacuum concentrator until dry. The metabolite pellets were detected by LC-MS.

\section{LC-MS analysis}

LC-MS analysis was performed by a Vanquish UHPLC system (Thermo Fisher) coupled with an Orbitrap Q Exactive HF-X mass spectrometer (Thermo Fisher) operating in the data-dependent acquisition (DDA) mode. Samples were injected onto a Hyperil Gold column $(100 \times 2.1 \mathrm{~mm}, 19 \mu \mathrm{m})$ by a 16 -min linear gradient at a flow rate of $0.3 \mathrm{~mL} / \mathrm{min}$. The positive polarity mode was eluented by eluent A (0.1\% FA in water) and eluent B (methanol), and the negative polarity mode were eluented by eluent $\mathrm{A}(5 \mathrm{mM}$ ammonium acetate, $\mathrm{pH}=$ 9.0) and eluent $B$ (methanol). The solvent gradient as follows: $2 \%$ B for $1.5 \mathrm{~min}, 2-100 \%$ B for $12 \mathrm{~min}, 100 \% \mathrm{~B}$ for $14 \mathrm{~min}, 100-2 \%$ for $14.1 \mathrm{~min}, 2 \%$ B for $16 \mathrm{~min}$. Q Exactive HF-X mass spectrometer was conducted on positive or negative polarity model with spray voltage of $3.2 \mathrm{kV}$, capillary temperature of $320^{\circ} \mathrm{C}$, sheath gas flow rate of 35 arb, and aux gas flow rate of 10 arb.

The raw data files analyzed by UHPLC-MS/MS were used by the compound discoverer 3.0 (CD 3.0, Thermo Fisher) to perform peak alignment, peak picking, and quantitation for each metabolite. The peaks were matched with the mzCloud (https://www.mzcloud.org/) and ChemSpider (http://www.chemspider.com/) database to get the accurate qualitative and relative quantitative results.

\section{Statistical analysis}

Statistical analysis was conducted using GraphPad Prism 6.01 (GraphPad Software, Inc., San Diego, CA). All data were expressed as the means \pm SEM. Differences between date means were determined using one-way ANOVA (Dunnett's t-test) and the two-tailed t-test. A $P<0.05$ is considered to be statistically significant. 


\section{Results}

\section{Induction of laminitis}

All horses were presented depressed appetite, profuse, and watery diarrhea $8-16 \mathrm{~h}$ after administration of oligofructose. Twenty hours later, the horse showed a reaction of weight-shifting and swelling over the coronary band, which were the pathological feature of laminitis. In addition, fecal $\mathrm{pH}$ was significantly reduced in the horse that treatment with oligofructose (Fig. 1).

\section{Effect of oligofructose treatment on lamellar tissue in hooves}

As shown in Fig. 2, no lesion was observed in normal hoof tissue sections demonstrated of the lamellar basement membrane. However, discontinuous loss and gaps in $H \& E$ and PAS staining was present in the lamellar basement membrane in laminitis horses after $48 \mathrm{~h}$ of oligofuctose dosing.

\section{Doppler ultrasonographic measurement}

As shown in Fig. 3a, in control horses, Doppler spectra were showed both high-resistance or low-resistance to nonresistance patterns, which were based on the overall degree of baseline elevation of end diastolic portion of the tracing. However, Doppler spectra of laminitis horses were characterized by low resistance blood flow with low peak systolic velocity (PSV) and low-end diastolic velocity (EDV). The horses suffered laminitis showed a PSV and EDV in doppler spectra decreased, indicating a decrease flow of vascular blood (Fig. 3b).

\section{Lactic acid, histamine, and LPS in serum}

As shown in Fig. 4a-c, treatment with oligofructose obviously increased the concentration of lactic acid, histamine, and LPS in serum.

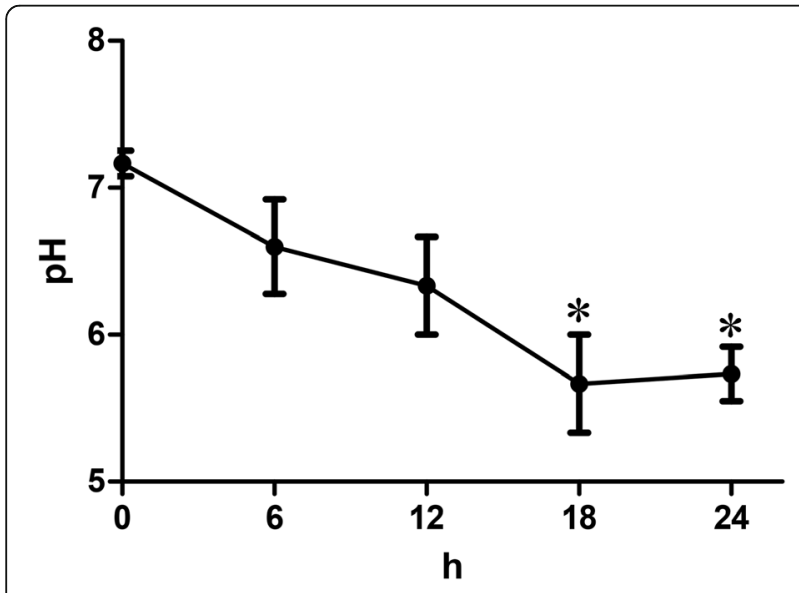

Fig. 1 Fecal $\mathrm{pH}$ and rectal temperature detection. a The fecal were collected and distilled with sterile PBS to detect pH at $6 \mathrm{~h}$ intervals in the $0-24 \mathrm{~h}$ oligofructose administration period. $P<0.05$ indicates a significant difference between the two groups
Composition of the hindgut bacterial community

Total 1607,927 reads, with an average of 80,396 reads from each sample, were detected using the V4 region of the bacterial $16 \mathrm{~S}$ ribosomal RNA (rRNA) gene amplified PCR from twenty fecal (10 from control and 10 from laminitis) samples. Rarefaction curves showed that the sampling depth had sufficient sequences to present the majority of bacterial diversity (Fig. S1). The bacterial community richness (observed species, chao 1, and ace) and diversity (shannon index and simpson index) were detected and showed that both community richness and diversity were significantly reduced after treatment with oligofructose (Fig. 5a-e). In addition, NMDS ordination performed on the Bray-Curtis dissimilarity showed that the bacterial community of fecal from healthy horses were separated from those that horses received oligofructose (Fig. 5f).

\section{Changes of hindgut bacterial community at the phylum level}

At the phyla level, these bacterial sequences obtained from all horses comprised 32 phyla, 28 of which shared in both groups. Among them, Firmicutes (control vs laminitis, $44.83 \%$ vs $59.17 \%$ ) and Bacteroidetes (control vs laminitis, $32.78 \%$ vs $22.84 \%$ ) were the most abundant phyla in the horse gut bacterial community. In addition, Proteobacteria (control vs laminitis, $5.39 \%$ vs $7.82 \%$ ), Kiritimatiellaeota (control vs laminitis $4.23 \%$ vs $1.03 \%$ ), Spirochaetes (control vs laminitis $3.21 \%$ vs $2.77 \%$ ), Euryarchaeota (control vs laminitis $1.67 \%$ vs $1.42 \%$ ), and Tenericutes (control vs laminitis $2.62 \%$ vs $1.19 \%$ ) were detected and the relative abundance $>1 \%$ in the horse intestines microbiota (Fig. 6a). To assess the changes of fecal microbiota, we compared the relative abundance of the bacterial abundant phyla between healthy and laminitis horses by T-test. The results showed that the relative abundance of Kiritimatiellaeota, Fibrobacteres, Tenericutes, Lentisphaerae, Elusimicrobia, Verrucomicrobia, and Planctomycetes were significantly reduced in intestinal microbiota from the laminitis horses when compared with those from the control horses (Fig. 6b).

\section{Changes of hindgut bacterial community at the genus levels}

At the genus level, these bacterial sequences detected from all the animals comprised 265 genera. As shown in Fig. 7a, the dominant genera were unidentified_Bacteroidales (control vs laminitis $3.95 \%$ vs $10.06 \%$ ), Lactobacillus (control vs laminitis, $0.20 \%$ vs $15.42 \%$ ), Succinicibrio (control vs laminitis, $3.76 \%$ vs $7.10 \%$ ), Anaerovibrio (control vs laminitis $0.50 \%$ vs $6.21 \%$ ), and Megasphaere (control vs laminitis, $0.45 \%$ vs $7.90 \%)$. Followed by unidentified_Prevotellaceae (control vs laminitis, $0.77 \%$ vs $3.40 \%$ ), Streptococcus (control vs laminitis $0.43 \%$ vs $2.96 \%$ ), Sharpea (control vs laminitis 

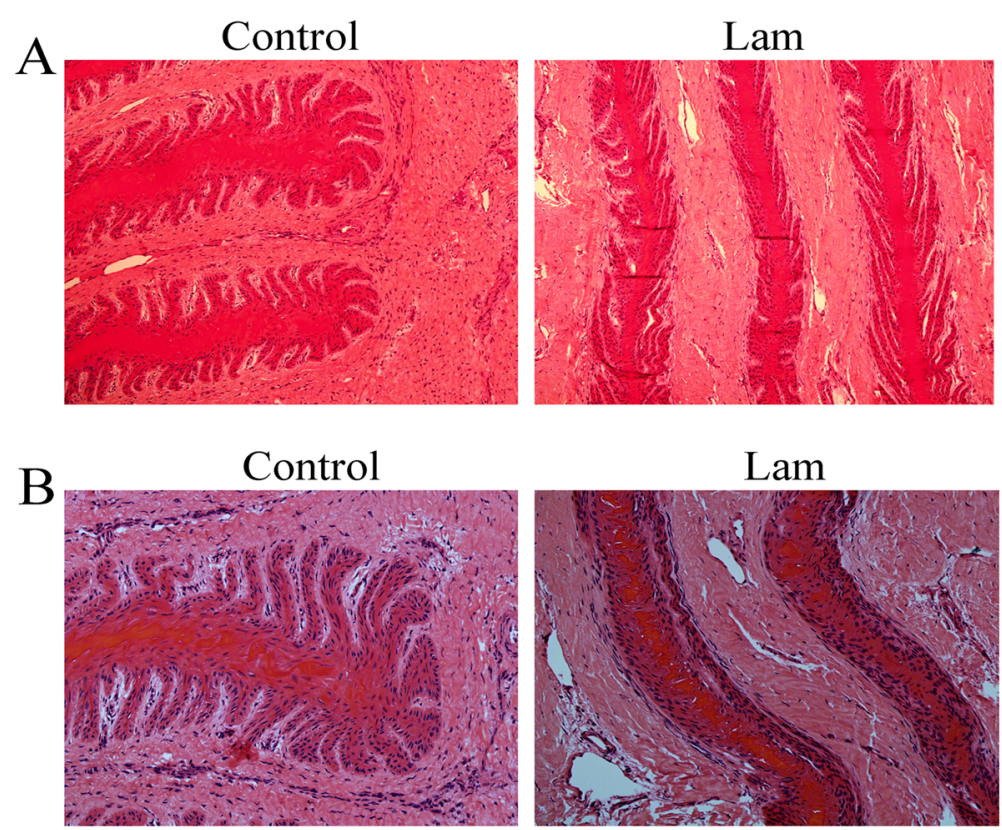

Fig. 2 Effect of oligofructose treatment on lamellar tissue in hooves. a H\&E staining. b PAS staining from hoof tissues between control and laminitis horses

0.01\% vs 3.09\%), Bifidobacterium (control vs laminitis, 0.1\% vs $1.21 \%$ ), and unidentified_Ruminococcaceae (control vs laminitis, $2.78 \%$ vs $2.29 \%$ ). T-test showed that the relative abundance of Lactobacillus, Megasphaera, and Allisonella were significantly increased, while the relative abundance of Fibrobacter, Phascolarctobacterium, Papillibacter, Alloprevotella, Candidatus_Soleaferrea, Oribactrium, Akkermansia, and Elusimicrobium were reduced in intestines microbiota from laminitis when compared to those from healthy horses (Fig. 7b).
Furthermore, the core genera shared by the healthy and laminitis horses were used to evaluate the link between unique bacterial microbiota and laminitis. As shown in Fig. 8a, most genera were shared between healthy and laminitis horse intestines microbiota. As showed core genera accounted for $80.50 \%$ of fecal microbiota on healthy horse and $90.43 \%$ on laminitis horse. These results suggested that changes in bacterial abundance and interactions among shared bacteria are more important for the development of laminitis. To
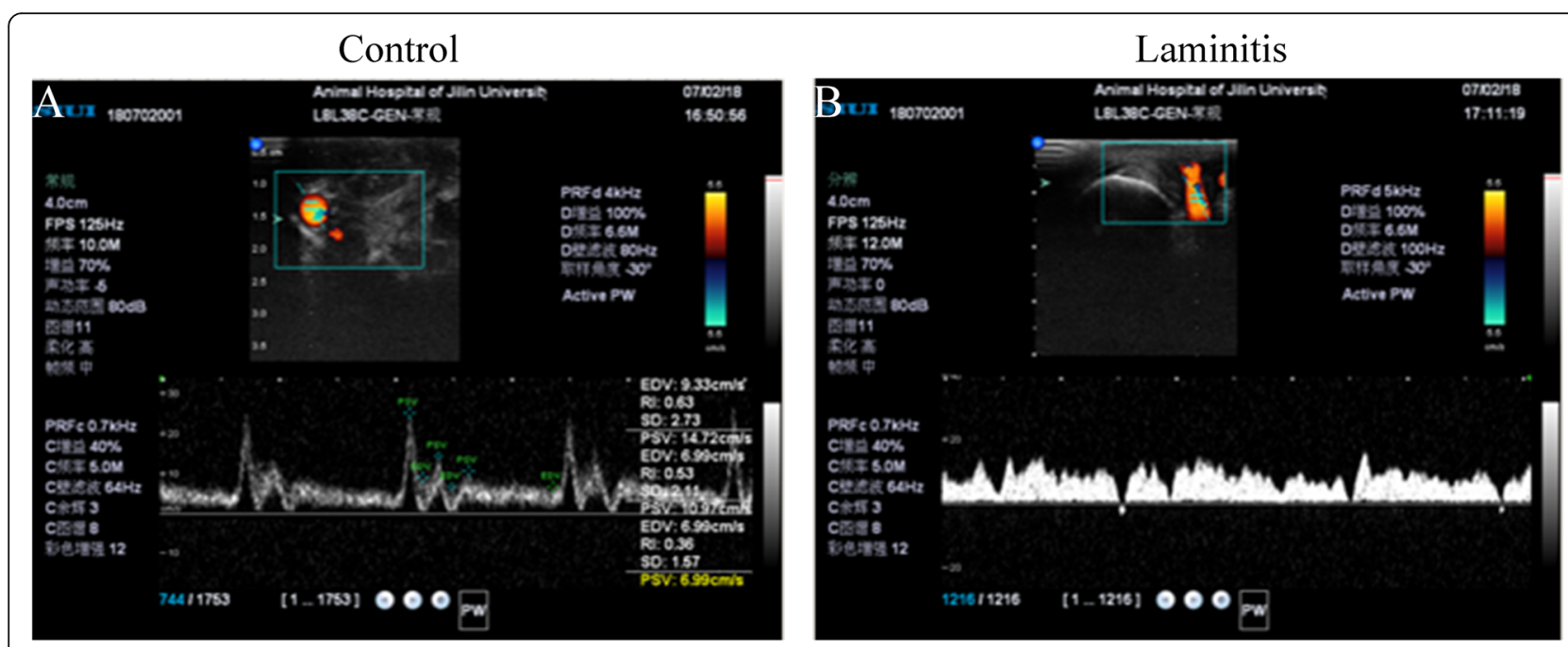

Fig. 3 Doppler Utrasonographic measurement. Doppler ultrasonographic measurement was performed on medial digital artery between control and laminitis horses 


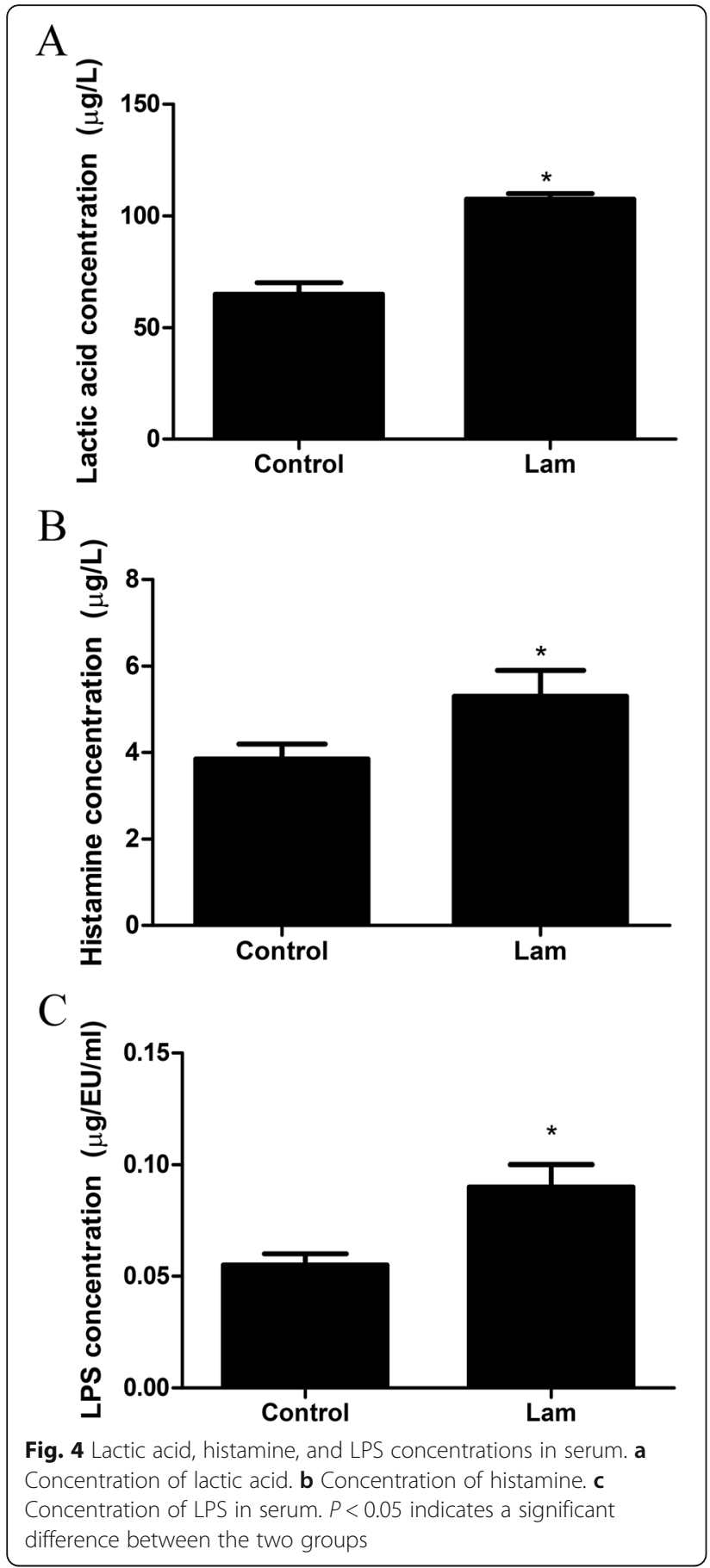

verify this hypothesis, we performed a biomarker analysis by a LEfSe and a cladogram generated from LEfSe analysis on the microbiota community of intestines. At the genus levels, the biomarkers with significant discriminative power were Lactobacillus, Megasphaera, Sharpea, and Streptococcs. At the species levels, the biomarkers with significantly discriminative power were Lactobacillus_gasseri, Prevotella_sp_DJF_CP65, Lactobacillus_ debrueckii, and Megasphaera_elsdenii (Fig. 8b-c). These results suggested that the bacterial that production of lactic acid play an important role in the development of equine laminitis induced by oligofructose.

\section{Changes of hindgut metabolites in horse suffering laminitis}

Principal component analysis (PCA) and Partial least squares-discriminant analysis (PLS-DA) were used to characterize the variations of the metabolic profiles between the control and laminitis group horses in the present study. As shown in Fig. 9a, the distribution of metabolites between control and laminitis horses were obviously separated, and PC 1 and PC 2 accounted for $41.47 \%$ and 15.31\% of the total variation, respectively. In addition, the OPLS-DA sores results also showed that there were significantly different and distinct metabolite compositions between control and laminitis group horses (Fig. 9b-c).

A total 485 metabolites, mainly fatty acids, lipids, amines, amino acids, organic acids, sugars, nucleosides and other metabolites were identified in the intestinal contents, 137 significantly different metabolites (VIP $>1, \mathrm{FC}>$ 2 , and $P<0.05), 53$ had higher concentrations, such as 10E,12Z-Octadecadienoic acid, asparagine, dihydrothymine, N3,N4-Dimethyl-L-arginine, glycine anhydride, creatine, caprolactam, histamine, N1-(1H-1,2,4-triazol-3-yl)2-hydroxybenzamide, tiglic acid, 5-Methylcytosine, in laminitis group than the control group. The remaining 84 metabolites were reduced in laminitis group, such as isophorone, D-(+)-Camphor, 4-Ethylbenzaldehyde, 3,5Dimethoxybenzoic acid, isohomovanillic acid, hydrocortisone 17-valerate, trans, trans-2,4-Heptadienal, phenylpropiolic acid, trans-3-Hexenoic acid, 2-Aminooctanoic acid, and methyl dihydrojasmonate (Table S3).

\section{Metabolic pathway analyses}

To further understand how multiple pathways changed after treated with oligofructose, analysis of the pathways with differential metabolites were conducted to the Kyoto Encyclopedia of Genes and Genomes (KEGG). As shown in Fig. 10, ABC transporters, glycerophospholipid metabolism, inflammatory mediator regulation of TRP channels, lysine degradation, vitamin digestion and absorption, and tyrosine metabolism were enriched in laminitis group compared with control group horses.

\section{Correlation between the gut microbiome and metabolome}

Correlation networks were conducted by means of the data concerning the most abundant taxa (differential genera top 10 between control and laminitis groups) and metabolites (differential metabolites top 20 between control and laminitis groups) created on Pearson's correlation coefficients $(\mid$ rho $\mid \geq 0.6)$ and $P \leq 0.05)$ in control 

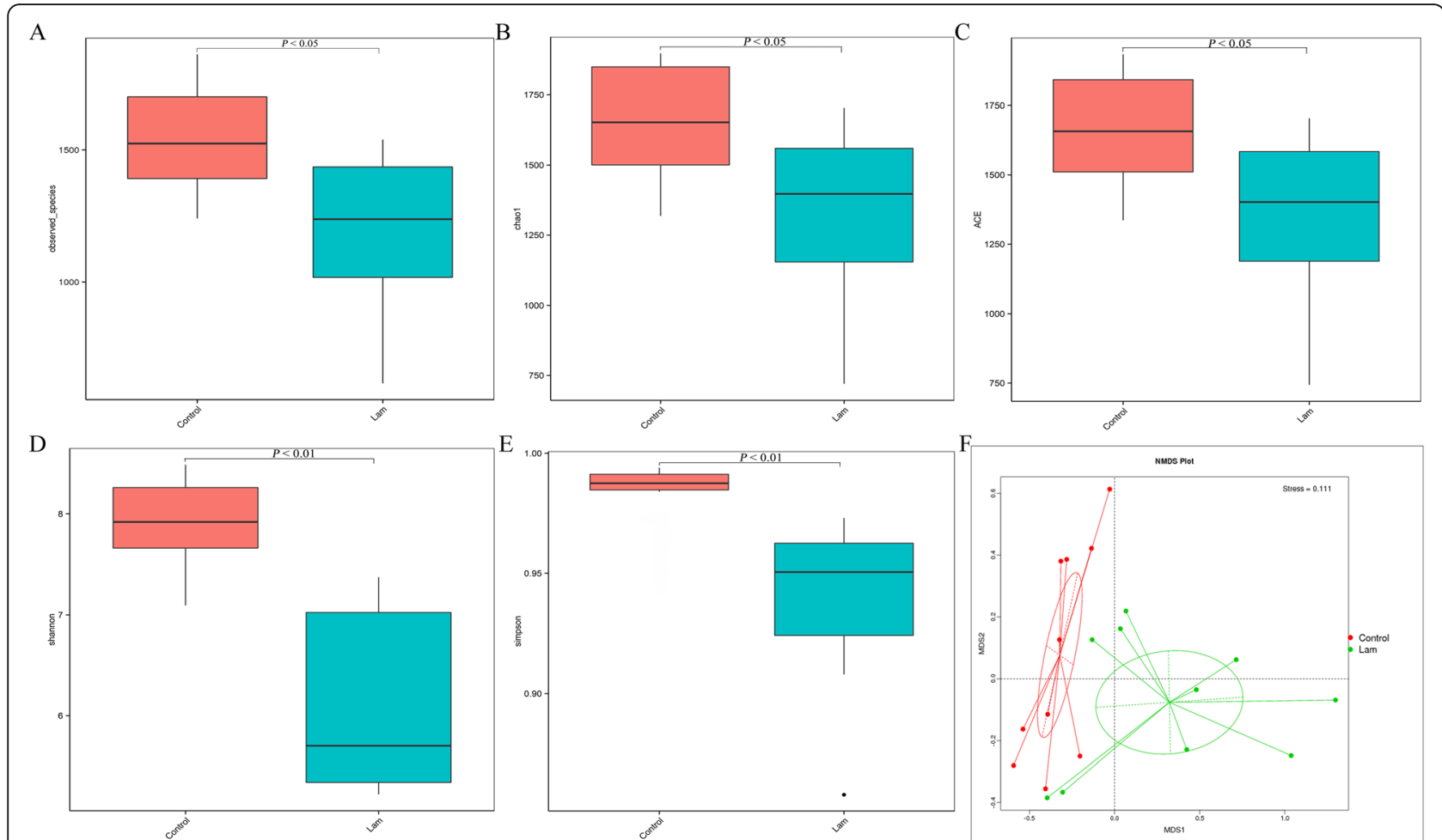

Fig. 5 The composition of the intestines bacterial community. Comparison of the microbiota richness in terms of (a) observes species, (b) chao 1, and (c) ace. Comparison of the microbiota diversity in terms of (d) shannon index, and (e) simpson index. f NMDS plot based on Bray-Curtis dissimilarity of fecal microbiota between healthy and laminitis horses. $P<0.05$ indicates a significant difference between the two groups

and laminitis groups (Fig. 11). Lactobacillus, Megasphaera, Sharpea, Bifidobacterium, and Mitsuokella were the most abundance of the geneus in the gut (Fig. 7a), thus the correlation networks were conducted on In addition to the dihydrothymine and N3, N4-Dimethyl-Larginine were positivity associated with Megasphagera, Lactobacillus and Megasphaera were both positively associated with 10E,12Z-Octadecadienoic acid, and asparagine, and were negatively associated with isophorone, 3,5-di (2-furylmethylidene)tetrahydro-2H-pyran-4one, D-(+)-Camphor, 4-Ethylbenzaldehyde, (3beta,9xi)3-(beta-D-Glucopyranosyloxy)-14-hydroxycard-20(22)enolide, 4,6,8-trihydroxy-7-methoxy-3-methyl-3,4-dihydro-1H-2-benzopyran-1-one, 2,6-Di-tert-butyl-1,4benzoquinone, $\gamma$-Nonanolactone, 3,5-Dimethoxybenzoic acid, isohomovanillic acid, hydrocortisone 17 -valerate, 2 ,

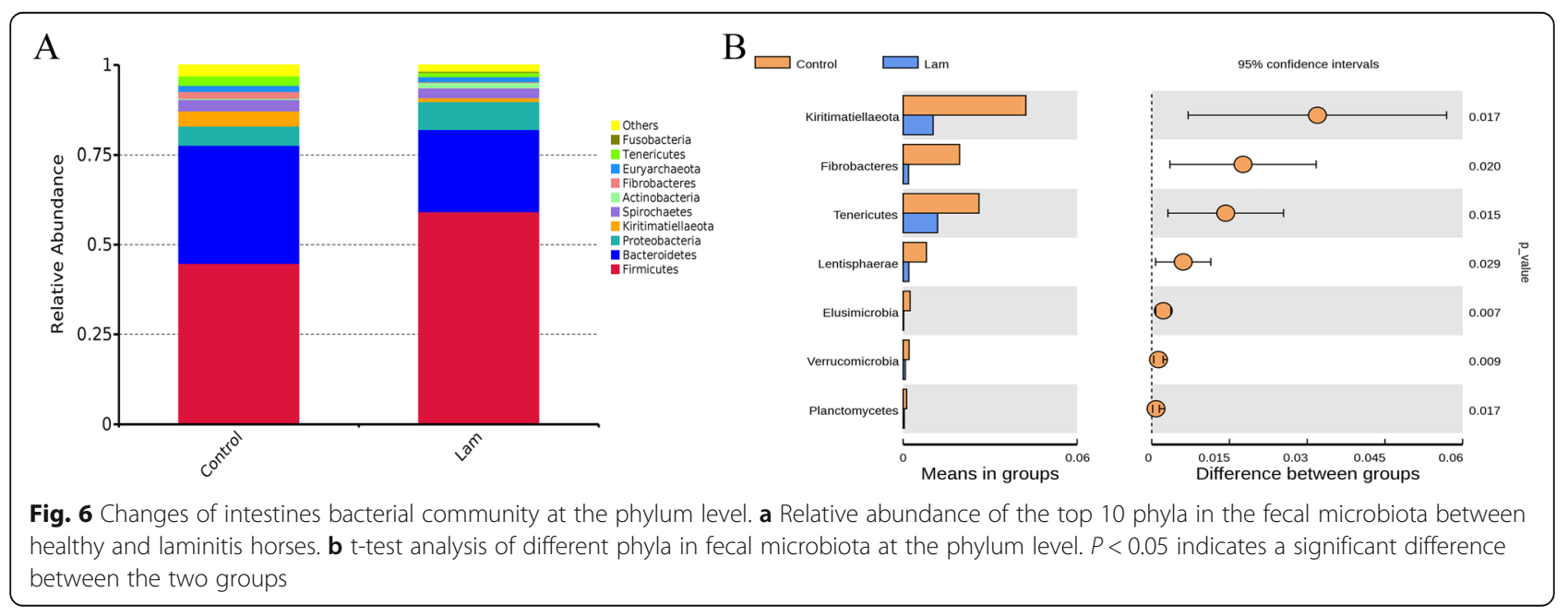



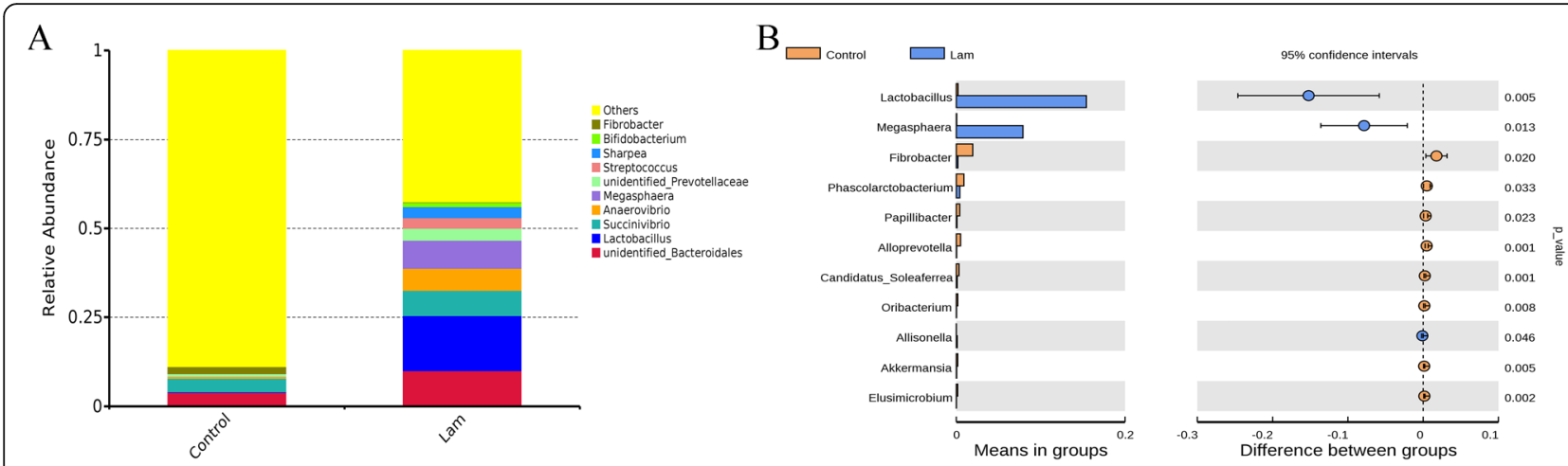

Fig. 7 Changes of intestines bacterial community at the genus level. a Relative abundance of the top 10 genus in the fecal microbiota between healthy and laminitis horses. $\mathbf{b}$ T-test analysis of different genus in fecal microbiota at the genus level. $P<0.05$ indicates a significant difference between the two groups

6-Dimethyl- $\gamma$-pyrone, trans,trans-2,4-Heptadienal, phenylpropiolic acid, and (S)-(-)-2-Hydroxyisocaproic acid. Sharpea was positively associated with asparagine, and negatively associated with 2,6-Di-tert-butyl-1,4-benzoquinone. Bifidobacterium was positively associated with 10E,12Z-Octadecadienoic acid. Mitsuokella was positively associated with 10E,12Z-Octadecadienoic.

\section{Discussion}

Laminitis is a kind of aseptic inflammation disease in the dermal layers of the hooves, and it causes great pain to the horse [21, 22]. Although the pathological mechanisms of laminitis are still unclear, the role of gut microbiota and its metabolites in equine laminitis is attracting more and more attention [18, 23]. Evidence indicated that the relative abundance of Lactobacillus and Streptococcus as well as the potentially pathogenic, including Veillonella and serratia, were increased in laminitis induced by oligofructose or cornstarch [23]. Others also showed that increased the relative abundance of streptococci of the Streptococcus bovis/equinus complex may be associated with the series of events which precede the onset of laminitis in the horse [6, 24]. In the present study, we found that the richness and diversity of bacterial community in hindgut were decreased in equine laminitis induced by oligofructose. In addition, the composition of the hindgut microbiota in the healthy and laminitis horses was also significantly different, as showed by the clear separation of sample points from two groups on the NMDS. Generally speaking, the changes were mainly reflected by increasing the relative abundance of genera of Lactobacillus, which was the major lactic acid producer. These results were consistent with the research of Milinovich et al., they suggested that Lactobacillus spp., were increased in oligofructose induced laminitis [6]. In addition, the relative abundance of genera Megasphaere, a key lactate utilizer [4, 25], also increased, while the relative abundance of Fibrobacter,
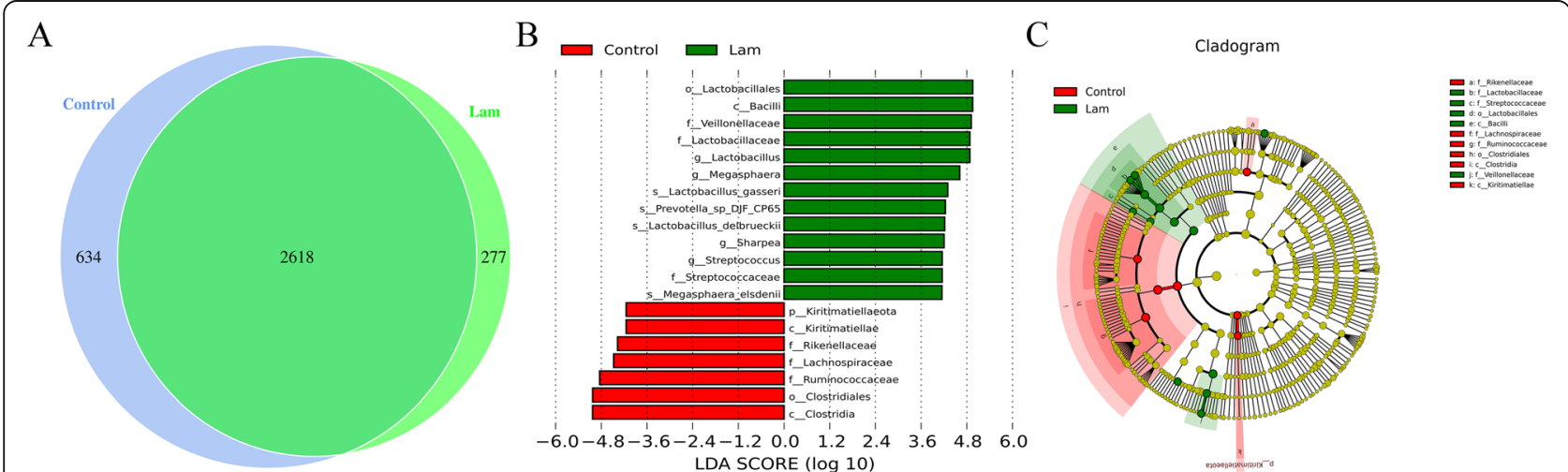

Fig. 8 Effect of oligofructose on the composition of the hingut microbial communities in horse at different taxonomic levels. a Venn diagram showing the number of core genera in fecal samples from healthy and laminitis horses. $\mathbf{b}$ Linear discriminant analysis (LDA) score derived from LEfSe analysis, showing the biomarker taxa (LDA score of $>4$ and a significance of $p<0.05$ calculated by the Wilcoxon signed-rank test) in fecal microbiota from healthy and laminitis horses. c Cladogram generated from LEfSe analysis showing the relationship between taxon at the levels of phylum, class, order, family, genus, and species. $P<0.05$ indicates a significant difference between the two groups 


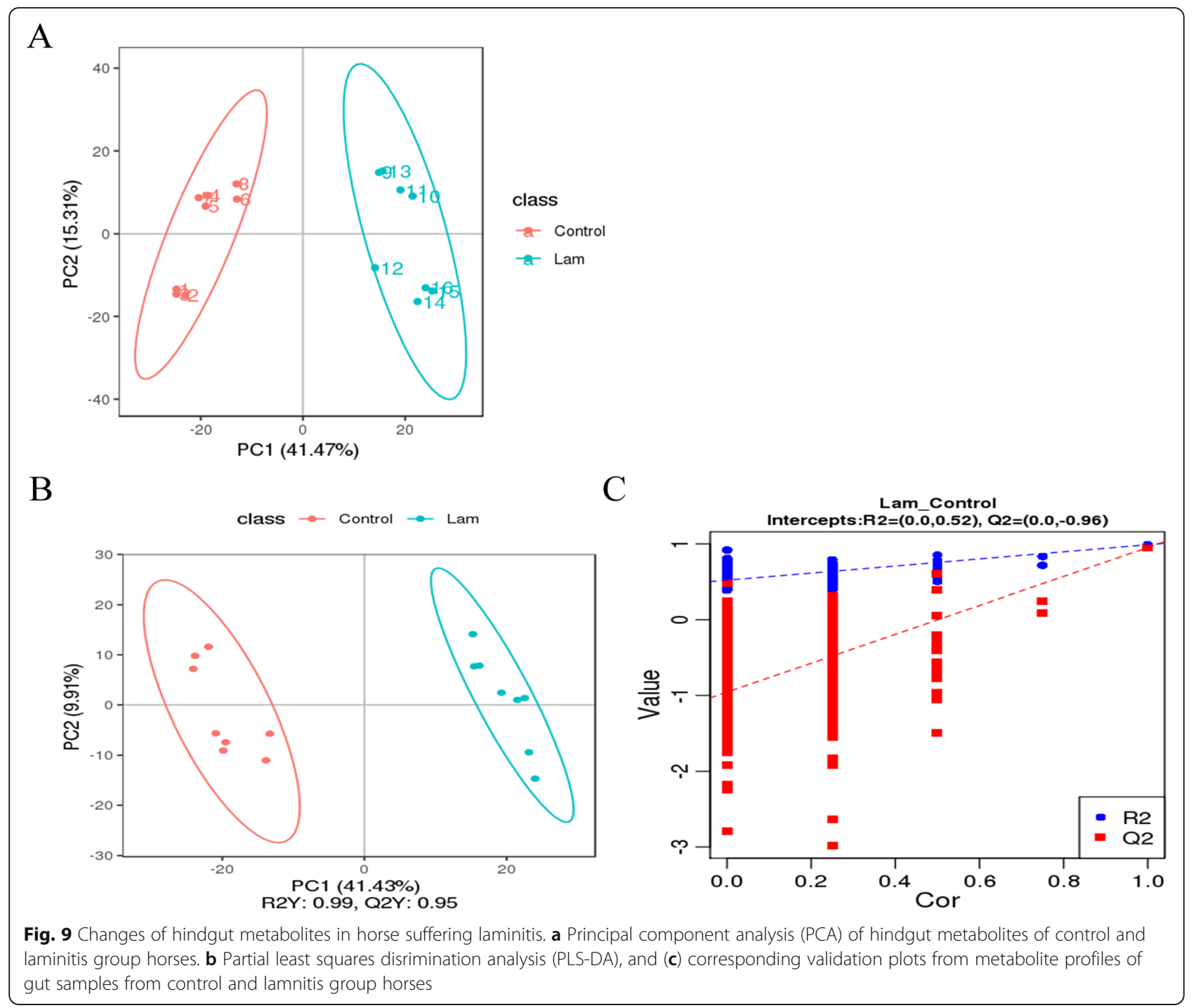

Phascolarctobacterium, Papillibacter, and Alloprevotella were significantly reduced. The others also indicated that increased the relative of Megasphaere has been associated with the lactic acid levels recover following starch induction conditions [4]. However, the levels of lactic acid also obviously higher in serum from laminitis horse than the level of lactic acid from healthy horses in the present study. These may be due to the concentrations of lactic acid produced was beyond the utilization ability of the Megasphaere. These suggested that the increased numbers of Lactobacillus and its metabolite lactate may be one of the most important factors to attribute the development of laminitis.

LPS, the cell wall component of gram-negative bacteria, has been considered to be one of the most important factors in the development of laminitis in horses. It suggested that once carbohydrate overload, a large number of gram-negative bacteria were killed and LPS was released due to the acidic environment which resulted from intaking high carbohydrate feedings. At the same time, the intestinal permeability was increased and then led to a large amount of LPS absorbed into the blood, causing local and systemic inflammatory responses, such as laminitis $[21,26]$. Other studies also proved that LPS can cause constriction of peripheral nerve blood vessels, activate the clotting system and cause damage to the hoof of cattle [27]. The present study also showed that the concentration of LPS in serum of laminitis horses were increased when it compared to healthy horses.

Another related theory of the aetiology of laminitis demonstrated that histamine was associated with the development of laminitis in both horses and cattle [28]. Histamine, a vasoactive agent, was shown to accumulated in the horses and cattle gut following by ingestion of high levels of carbohydrates, and led to damage the hoof through circulation $[11,29,30]$. The present study 


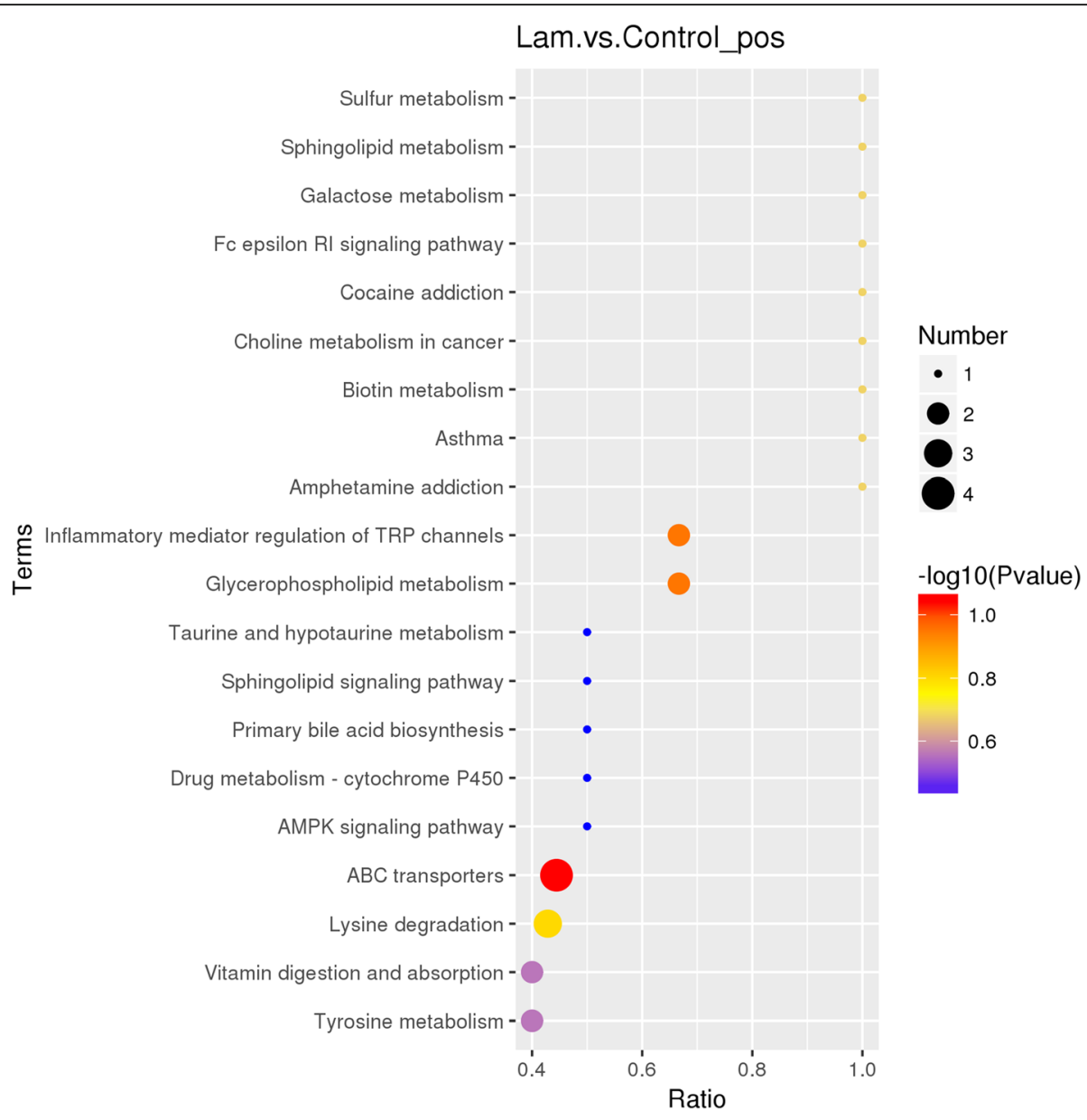

Fig. 10 Metabolic pathway analyses. Pathways associated with the metabolites identified in control and lamnitis group horses. The larger size represents higher pathway enrichment, and darker color represents higher pathway impact values

indicated that the concentration of histamine was significantly increased in the serum in laminitis horse. In addition, some bacterial strains, such as Lactobacillus [31], have been identified as being able to secrete histamine due to the decarboxylation of histidine through the enzyme histidine decarboxylase activity. The results also showed that the relative abundance of Lactobacillus. was obviously increased in the equine intestines bacterial community during laminitis induced by oligofructose. Metabolomics analysis revealed that in addition to the LPS, lactic acid, and histamine, more than 50 metabolitic concentrations were increased in intestinal contents from laminitis horse compared to the control horse. 10E,12Z-Octadecadienoic acid and asparagine not only increased in the horse treated with oligofructose, but also positively associated with the abundance of Lactobacillus and Megasphaera. Previous studies showed that treatment of 9-hydroxy-10E,12Z-Octadecadienoic acid disrupted epidermal barrier in human keratinocytes [32]. Dihydrothymine is an intermediate in liver pyrimidine catabolism. Studies in animals indicated that changes of pyrimidine metabolism with increased hepatic lipid [33, 34]. Asparagine is a non-essential amino acid in normal cells, as cells are able to synthesize asparagine from other amino acid, such as glutamate/glutamine and aspartate, though transaminases and asparagine synthetase [35]. Studies suggested that depletion of asparagine was associated with improved outcomes in ALI [35]. In addition, obese phenotype was associated with the increase the concentration of asparagine, and others also showed that asparagine is negatively correlated to unhealthy metabolic conditions [36-38]. Arginine is not considered to be an non-essential amino acid for healthy humans, however, it is classified as a semi-essential amino acid with in view of dietary requirement [39]. Ttest showed the abundance of Lactobacillus, and Megasphaera were significantly increased in laminitis group horses when compared to the control group, and correlation analysis showed the Lactobacillus and Megasphaera both were positively associated with the 10E, 12Z-Octadecadienoic acid, and asparagine. Numbers of evidence suggested that the metabolic alterations 


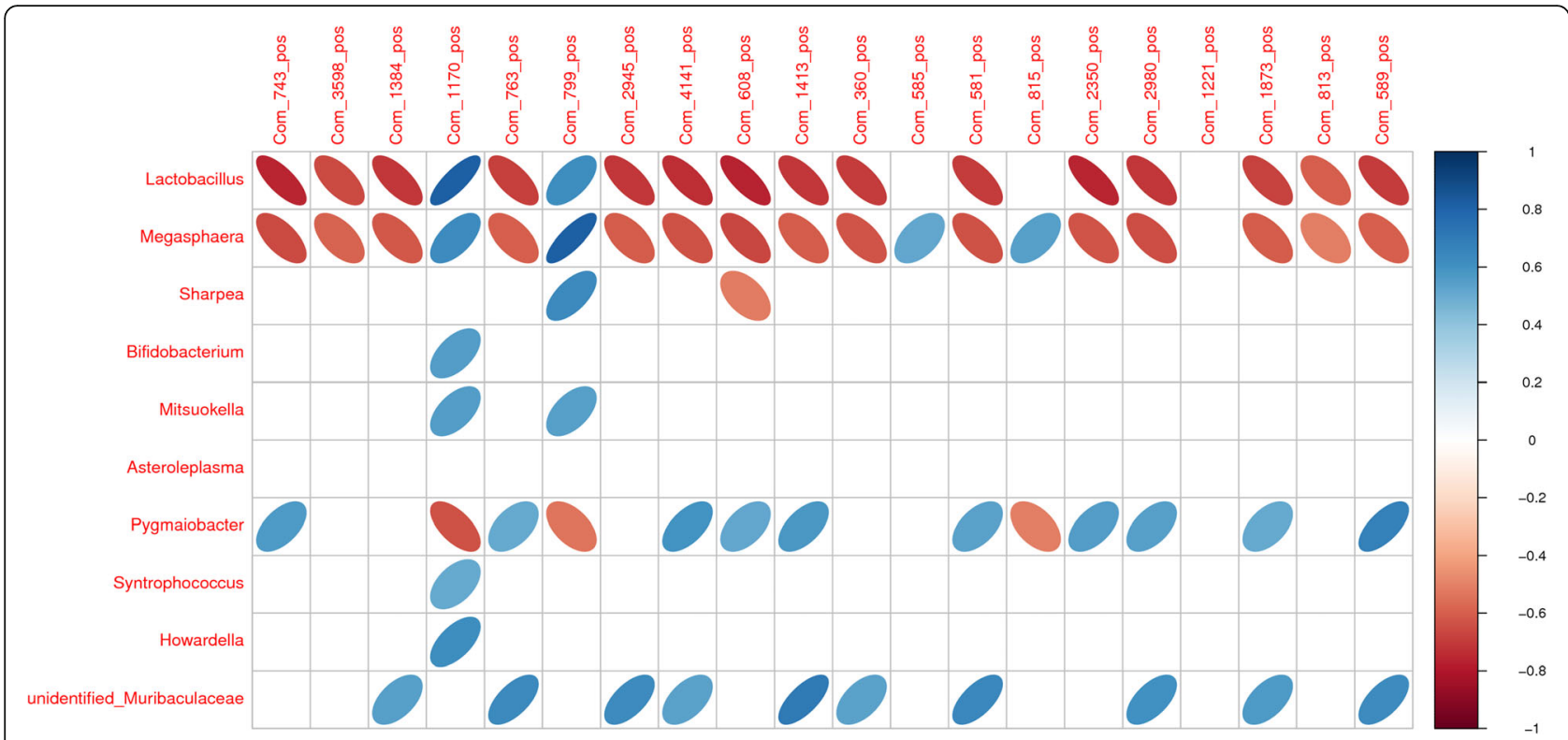

Fig. 11 Correlation between the gut microbiota and metabolome. The spearmen correlation network between gut microbiota and metabolome in control and lamnitis group horses

associated with gut bacterial community disturbance are important biomarkers that indicate the health of human and animals [40-42]. These results suggested that increase of Lactobacillus and Megasphaera, as well as 10E, 12Z-Octadecadienoic acid, asparagine, dihydrothymine, and N3,N4-Dimethyl-L-arginine may be as an auxiliary diagnostic indicator for laminitis in horses.

\section{Conclusions}

In conclusion, we found that oligofructose treatment altered the pattern of hindgut normal bacterial community and metabolites, showed by increased relative abundance of the Lactobacillus. Megasphaera and levels of lactic acid, histamine, LPS, 10E,12Z-Octadecadienoic acid, asparagine, dihydrothymine, and N3,N4-Dimethyl-L-arginine, which may be associated with the damage of laminar tissues of horses. Hence, targeting intestine microbiota and its metabolites may be an important target for preventing equine laminitis.

\section{Supplementary Information}

The online version contains supplementary material available at https://doi. org/10.1186/s12917-020-02686-9.

Additional file 1: Table S1. Relative abundance of all phyla in the fecal microbiota between healthy and laminitis horses. (XLS 28 kb)

Additional file 2: Table S2. Relative abundance of all genus in the fecal microbiota between healthy and laminitis. (XLS $28 \mathrm{~kb}$ )

Additional file 3: Table S3. Differential metabolite screening results (XLS $47 \mathrm{~kb}$ )

Additional file 4: Figure S1. A rarefaction curve was used to analyze sampling depth to the majority of bacterial diversity.

\section{Abbreviations}

LPS: Lipopolysaccharide; POA: Post-oligofructose administration; H\&E: Hematoxylin and Eosin; PAS: Periodic acid-Schiff; NMDS: nonmetric multidimensional scaling; LEfse: linear discriminant analysis (LDA) effect size; DDA: data-dependent acquisition; PSV: Peak systolic velocity; EDV: End diastolic velocity; PCA: Principal component analysis; PLS-DA: Partial least squares-discriminant analysis; KEGG: Kyoto Encyclopedia of Genes and Genomes

\section{Acknowledgments}

The authors with to thank all of the breeders for their contributions.

\section{Authors' contributions}

MT and X H performed and established the model of laminitis and the sample collection and preparation of the manuscript. J $Y$ and J G performed the analysis of gut microbiota and metabolites. $S \mathrm{~L}$ permed the detection of blood index. N Z performed the supervised the research project and reviewed the paper. Y F performed the design of the study. All authors read and approved the final manuscript.

\section{Funding}

This work was supported by a grant from the National Natural Science Foundation of China (Nos. 31472248, 31772812).

\section{Availability of data and materials}

The dataset is available from the corresponding author on reasonable request.

\section{Ethics approval and consent to participate}

The full proposal was reviewed by the Institutional Animal Care and Use Committee (IACUC) of Jilin University ethics committee, which approved the animal care and use permit license. All experiments comply with the manual of the care and use of laboratory animals published by the US National Institutes of Health.

\section{Consent for publication} Not applicable. 


\section{Competing interests}

None of the authors have a financial interest in any of the products, devices, or Materials mentioned in this manuscript. The authors declare that they have no conflicts of interest.

\section{Received: 3 October 2020 Accepted: 19 November 2020} Published online: 06 January 2021

\section{References}

1. Fages A, Hanghoj K, Khan N, Gaunitz C, Seguin-Orlando A, Leonardi M, Mccrory Constantz C, Gamba C, Al-Rasheid KAS, Albizuri S, Alfarhan AH, Allentoft M, Alquraishi S, Anthony D, Baimukhanov N, Barrett JH, Bayarsaikhan J, Benecke N, Bernaldez-Sanchez E, Berrocal-Rangel L, Biglari F, Boessenkool S, Boldgiv B, Brem G, Brown D, Burger J, Crubezy E, Daugnora L, Davoudi H, de Barros Damgaard P, de Los Angeles de Chorro YdV-CM, Deschler-Erb S, Detry C, Dill N, do Mar Oom M, Dohr A, Ellingvag S, Erdenebaatar D, Fathi H, Felkel S, Fernandez-Rodriguez C, Garcia-Vinas E, Germonpre M, Granado JD, Hallsson JH, Hemmer H, Hofreiter M, Kasparov A, Khasanov M, Khazaeli R, Kosintsev P, Kristiansen K, Kubatbek T, Kuderna L, Kuznetsov P, Laleh H, Leonard JA, Lhuillier J, Liesau von Lettow-Vorbeck C, Logvin A, Lougas L, Ludwig A, Luis C, Arruda AM, Marques-Bonet T, Matoso Silva R, Merz V, Mijiddorj E, Miller BK, Monchalov O, Mohaseb FA, Morales A, Nieto-Espinet A, Nistelberger H, Onar V, Palsdottir AH, Pitulko V, Pitskhelauri K, Pruvost M, Rajic Sikanjic P, Rapan Papesa A, Roslyakova N, Sardari A, Sauer E, Schafberg R, Scheu A, Schibler J, Schlumbaum A, Serrand N, SerresArmero A, Shapiro B, Sheikhi Seno S, Shevnina I, Shidrang S, Southon J, Star B, Sykes N, Taheri K, Taylor W, Teegen WR, Trbojevic Vukicevic T, Trixl S, Tumen D, Undrakhbold S, Usmanova E, Vahdati A, Valenzuela-Lamas S, Viegas C, Wallner B, Weinstock J, Zaibert V, Clavel B, Lepetz S, Mashkour M, Helgason A, Stefansson K, Barrey E, Willerslev E, Outram AK, Librado P, Orlando L. Tracking Five Millennia of Horse Management with Extensive Ancient Genome Time Series. Cell. 2019;177:1419-35 e1431..

2. Lippold S, Matzke NJ, Reissmann M, Hofreiter M. Whole mitochondrial genome sequencing of domestic horses reveals incorporation of extensive wild horse diversity during domestication. BMC Evol Biol. 2011;11:328.

3. Mungall BA, Pollitt CC. Zymographic analysis of equine laminitis. Histochem Cell Biol. 1999;112:467-72.

4. Biddle AS, Black SJ, Blanchard JL. An in vitro model of the horse gut microbiome enables identification of lactate-utilizing bacteria that differentially respond to starch induction. PLoS One. 2013;8:e77599.

5. Peloso JG, Cohen ND, Walker MA, Watkins JP, Gayle JM, Moyer W. Casecontrol study of risk factors for the development of laminitis in the contralateral limb in Equidae with unilateral lameness. J Am Vet Med Assoc. 1996;209:1746-9.

6. Milinovich GJ, Trott DJ, Burrell PC, van Eps AW, Thoefner MB, Blackall LL, Al Jassim RA, Morton JM, Pollitt CC. Changes in equine hindgut bacterial populations during oligofructose-induced laminitis. Environ Microbiol. 2006; 8:885-98.

7. Weese JS, Holcombe SJ, Embertson RM, Kurtz KA, Roessner HA, Jalali M, Wismer SE. Changes in the faecal microbiota of mares precede the development of post partum colic. Equine Vet J. 2015;47:641-9.

8. Weese JS, Staempfli HR, Prescott JF. A prospective study of the roles of clostridium difficile and enterotoxigenic Clostridium perfringens in equine diarrhoea. Equine Vet J. 2001;33:403-9.

9. Al Jassim RA, Andrews FM. The bacterial community of the horse gastrointestinal tract and its relation to fermentative acidosis, laminitis, colic, and stomach ulcers. Vet Clin North Am Equine Pract. 2009;25:199-215.

10. Milinovich GJ, Trott DJ, Burrell PC, Croser EL, Al Jassim RA, Morton JM, van Eps AW, Pollitt CC. Fluorescence in situ hybridization analysis of hindgut bacteria associated with the development of equine laminitis. Environ Microbiol. 2007;9:2090-100.

11. Garner MR, Flint JF, Russell JB. Allisonella histaminiformans gen. nov., sp nov. A novel bacterium that produces histamine, utilizes histicline as its sole energy source, and could play a role in bovine and equine laminitis. Syst Appl Microbiol. 2002;25:498-506.

12. Bailey SR, Rycroft A, Elliott J. Production of amines in equine cecal contents in an in vitro model of carbohydrate overload. J Anim Sci. 2002;80:2656-62.

13. Pollitt CC, Daradka M. Equine laminitis basement membrane pathology: loss of type IV collagen, type VII collagen and laminin immunostaining. Equine Vet J Suppl. 1998:139-44..
14. Kyaw-Tanner M, Pollitt CC. Equine laminitis: increased transcription of matrix metalloproteinase-2 (MMP-2) occurs during the developmental phase. Equine Vet J. 2004;36:221-5.

15. Garner HE, Hutcheson DP, Coffman JR, Hahn AW, Salem C. Lactic acidosis: a factor associated with equine laminitis. J Anim Sci. 1977;45:1037-41.

16. Pollitt CC. Basement membrane pathology: a feature of acute equine laminitis. Equine Vet J. 1996;28:38-46.

17. Van Eps A, Pollitt CC. Equine laminitis induced with oligofructose. Equine Vet J. 2006:38:203-8

18. Milinovich GJ, Burrell PC, Pollitt CC, Klieve AV, Blackall LL, Ouwerkerk D, Woodland E, Trott DJ. Microbial ecology of the equine hindgut during oligofructose-induced laminitis. Isme J. 2008;2:1089-100.

19. Karikoski NP, McGowan CM, Singer ER, Asplin KE, Tulamo RM, PattersonKane JC. Pathology of natural cases of equine Endocrinopathic laminitis associated with Hyperinsulinemia. Vet Pathol. 2015;52:945-56.

20. Wongaumnuaykul S, Siedler C, Schobesberger H, Stanek C. Doppler sonographic evaluation of the digital blood flow in horses with laminitis or septic pododermatitis. Pferdeheilkunde. 2006;22:472-3.

21. van Eps AW, Pollitt CC. Equine laminitis: cryotherapy reduces the severity of the acute lesion. Equine Vet J. 2004;36:255-60.

22. Zhang RY, Jin W, Feng PF, Liu JH, Mao SY. High-grain diet feeding altered the composition and functions of the rumen bacterial community and caused the damage to the laminar tissues of goats. Animal. 2018;12:2511-20.

23. Moreau MM, Eades SC, Reinemeyer CR, Fugaro MN, Onishi JC. Illumina sequencing of the $\mathrm{V} 4$ hypervariable region $16 \mathrm{~S}$ rRNA gene reveals extensive changes in bacterial communities in the cecum following carbohydrate oral infusion and development of early-stage acute laminitis in the horse. Vet Microbiol. 2014;168:436-41.

24. Milinovich GJ, Trott DJ, Burrell PC, Croser EL, Al Jassim RAM, Morton JM, van Eps AW, Pollitt CC. Fluorescence in situ hybridization analysis of hindgut bacteria associated with the development of equine laminitis (vol 9, pg 2090, 2007). Environ Microbiol. 2008;10:2901.

25. Marounek M, Bartos S. Interactions between rumen amylolytic and lactateutilizing bacteria in growth on starch. J Appl Bacteriol. 1987;63:233-8.

26. Toth F, Frank N, Chameroy KA, Boston RC. Effects of endotoxaemia and carbohydrate overload on glucose and insulin dynamics and the development of laminitis in horses. Equine Vet J. 2009;41:852-8.

27. Boosman $\mathrm{R}$, Mutsaers $\mathrm{CW}$, Klarenbeek $\mathrm{A}$. The role of endotoxin in the pathogenesis of acute bovine laminitis. Vet Q. 1991;13:155-62.

28. Irwin LN, Mitchell GE Jr, Tucker RE, Schelling GT. Histamine, tyramine, tryptamine and electrolytes during glucose induced lactic acidosis. J Anim Sci. 1979;48:367-74.

29. Takahashi K, Young BA. Effects of grain overfeeding and histamine injection on physiological responses related to acute bovine laminitis. Nihon Juigaku Zasshi. 1981;43:375-85

30. Mgasa MN. Bovine pododermatitis aseptica diffusa (laminitis) aetiology, pathogenesis, treatment and control. Vet Res Commun. 1987;11:235-41.

31. Gao CX, Ganesh BP, Shi ZC, Shah RR, Fultz R, Major A, Venable S, Lugo M, Hoch K, Chen XW, Haag A, Wang TC, Versalovic J. Gut microbe-mediated suppression of inflammation-associated Colon carcinogenesis by luminal histamine production. Am J Pathol. 2017;187:2323-36.

32. Chiba T, Nakahara T, Fujishima K, Hashimoto-Hachiya A, Furue M. Epidermal barrier disruption by 9-hydroxy-10E,12Z-octadecadienoic acid in human keratinocytes. J Dermatol. 2018;45:746-7.

33. Le TT, Ziemba A, Urasaki Y, Hayes E, Brotman S, Pizzorno G. Disruption of uridine homeostasis links liver pyrimidine metabolism to lipid accumulation. J Lipid Res. 2013;54:1044-57.

34. Khusial RD, Cioffi CE, Caltharp SA, Krasinskas AM, Alazraki A, Knight-Scott J, Cleeton R, Castillo-Leon E, Jones DP, Pierpont B, Caprio S, Santoro N, Akil A, Vos MB. Development of a plasma screening panel for pediatric nonalcoholic fatty liver disease using metabolomics. Hepatol Commun. 2019;3:1311-21.

35. Marini BL, Perissinotti AJ, Bixby DL, Brown J, Burke PW. Catalyzing improvements in ALL therapy with asparaginase. Blood Rev. 2017;31:328-38.

36. Chashmniam S, Hashemi MN, Nobakht Mothlagh Ghoochani BF, SafariAlighiarloo N, Khamseh ME. The metabolome profiling of obese and nonobese individuals: Metabolically healthy obese and unhealthy non-obese paradox. Iran J Basic Medi Sci. 2020;23:186-94.

37. Cheng SS, Rhee EP, Larson MG, Lewis GD, McCabe EL, Shen DX, Palma MJ, Roberts LD, Dejam A, Souza AL, Deik AA, Magnusson M, Fox CS, O'Donnell CJ, Vasan RS, Melander O, Clish CB, Gerszten RE, Wang TJ. Metabolite 
profiling identifies pathways associated with metabolic risk in humans Circulation. 2012;125:2222-U2132.

38. Guevara-Cruz M, Vargas-Morales JM, Mendez-Garcia AL, Lopez-Barradas AM, Granados-Portillo O, Ordaz-Nava G, Rocha-Viggiano AK, Gutierrez-Leyte CA, Medina-Cerda E, Rosado JL, Morales JC, Torres N, Tovar AR, Noriega LG. Amino acid profiles of young adults differ by sex, body mass index and insulin resistance. Nutr Metab Cardiovasc Dis. 2018;28:393-401.

39. Morris SM Jr. Arginine metabolism revisited. J Nutr. 2016;146:2579S-86S.

40. Ticinesi A, Nouvenne A, Tana C, Prati B, Meschi T. Gut microbiota and microbiotarelated metabolites as possible biomarkers of cognitive aging. Reviews on Biomarker Studies in Aging and Anti-Aging Research. 2019;1178:129-54.

41. Aragones G, Gonzalez-Garcia S, Aguilar C, Richart C, Auguet T. Gut microbiota-derived mediators as potential markers in nonalcoholic fatty liver disease. Biomed Res Int. 2019.

42. Carbonero F, Mayta A, Bolea M, Yu JZ, Lindeblad M, Lyubimov A, Neri F, Szilagyi E, Smith B, Halliday L, Bartholomew A. Specific members of the gut microbiota are reliable biomarkers of irradiation intensity and lethality in large animal models of human health. Radiat Res. 2019;191:107-21.

\section{Publisher's Note}

Springer Nature remains neutral with regard to jurisdictional claims in published maps and institutional affiliations.

- fast, convenient online submission

- thorough peer review by experienced researchers in your field

- rapid publication on acceptance

- support for research data, including large and complex data types

- gold Open Access which fosters wider collaboration and increased citations

- maximum visibility for your research: over $100 \mathrm{M}$ website views per year

At BMC, research is always in progress.

Learn more biomedcentral.com/submissions 